\title{
Mathematical Modelling and Simulation of liquid steel flow phenomena and temperature distribution in an optimized tundish design of a continuous caster
}

\author{
Ankit Bhardwaj \\ Jindal Stainless Limited, Industrial Area, O.P. Jindal Marg, Hisar-125011, Haryana, India
}

\begin{abstract}
The objective of this study was to analyze the fluid flow of molten steel in a continuous casting tundish using numerical simulations for better inclusion floatation and its separation. The tundish geometry was designed using Autodesk FUSION 360 and the analysis were performed on ANSYS FLUENT. The investigations were done on steady-state as well as transient conditions. To scale back vortexing and turbulence within the tundish, turbo stoppers and flow modulators, e.g. dam and weirs were placed for an optimized and efficient flow inside the tundish and its behavior on the spacious flow structure was explored. The strategic placements of the flow modifiers produced higher turbulence in the recess region of the tundish resulting in better turbulent flow withinside the inlet region of the tundish. Thereby a more homogeneous fluid flow is formed with better conditions for particle separation. Analysing the flow behavior we have determined the inclusion floatation using particle tracking method form dense discrete phase modelling along with multiphase eulerian-lagragian model. Reduction in dead volumes was achieved in the spatial flow due to better intermixing which further reduced the metal loss and increased the yield of the tundish using the fluid flow analysis. Analyzing eddy formations in the spatial geometry of the tundish structure made it easy to evenly distributes the flow-induced shear. This determined the lesser turbulence on the free surface of the steel flow resulting in less reduction of the liquid steel surface.
\end{abstract}

\section{Introduction}

Steel production underwent a drastic change in the middle of the 20th century when ingot casting was replaced by the continuous casting process. In ingot casting, the tip of each ingot has to be cut off after being removed from the mould, which leaves behind scrap metal. However, in continuous casting, this cut only needs to be made at the beginning and end of each sequence, where tons of steel are cast thus resulting in significantly less waste material. In addition, the shape of the fused strands is much closer to the shape of the final rolled product, resulting in improved performance, higher quality and greater cost efficiency over previous steelmaking processes. Unsurprisingly, 95 percent of steel today is made using the continuous casting process. In the continuous casting process, the molten-refined steel is often delivered to the caster in ladles of 50 metric tonne capacities. The molten steel is then teemed into a tundish. The first solid steel is formed in the watercooled copper mould and the formed strands are then further pulled out of the mould with driven rollers. Steel flow, temperature distribution and non-metallic particles concentration in the ladle, tundish and mould are of decisive importance for the quality of the steel.

-Corresponding author: ankit.bhardwaj@,jshl.in
The tundish behaves as a reserve and supplier of molten steel. Main responsibility of tundish is to enhance the inclusion floatation and separation. In composition adjustment in casting process, tundish plays a crucial role. The molten steel flow behavior is monitored closely for better efficiency and optimization in fluid flow model else, it may reduce the quality of steel produced.

The tundish performs various functions in the continuous casting process by playing the key role of a distinctive unit where critical operations such as melt temperature control, composition, melt flow control, etc. are performed to facilitate non-metallic inclusion separations. For the sake of high-quality steel manufacturing, there has been a huge demand for better technologies to remove inclusions from liquid steel thus fulfilling the increasing customer demand. Inclusions are nothing but sulphides, silicates and aluminium particles that are made during the casting process. If these inclusions are sufficiently large enough and get trapped in the molten steel, they might result in unwanted material properties and many sorts of defects. The dynamics of molten steel flow is an important factor in analyzing and controlling the inclusion floatation. The study of inclusion separation at an industrial level is quite difficult due to high temperature and lack of laser optical instruments. Hence, using mathematical modelling and running numerical simulations with the 
help of some tools of Computational fluid dynamics (CFD) we had computed the fluid flow phenomenon inside the spatial flow structure of the tundish. This further helped in strategic placement of turbo-stoppers and flow modifiers such as dams and weirs that resulted in producing an optimized and efficient homogenous fluid flow.

Regarding tundish, two types of flow condition arise,

One may be the case of Steady-state casting, where the mass of liquid steel in the tundish is conserved, i.e. constant. Here, the mass flow through the shroud is equal to the mass flow coming out through the submerged entry nozzle into the mould. Hence, the liquid steel level inside the tundish is constant.

Another one might be the case of Transient-state casting where the mass of liquid steel inside tundish varies with time. It depicts either the filling or draining of the fluid inside the tundish either with first or last ladle respectively or the process of ladle change when almost $75 \%$ of steel level sinks. Practically, hot slabs and billets during transient casting generally have a significantly higher number of surface defects than those made from steady state casting.

Important factors that manipulate the fluid flow inside the tundish are geometry, location of shrouds, turbo stoppers, flow modulators, along with mass flow rate and steel level in the tundish. In literature, various flow phenomena have been discussed such as:

- Fluctuating flow components generation from transient state casting that can lead to an increase in slab impurities. Splashing of molten steel and entrapment of gas bubbles in the filling up of a tundish is a classic illustration of such a case [1].

- For particles having a diameter below $50 \mu \mathrm{m}$, a significant high frequency of small scaled eddies may disturb the particle separation [2].

- Counter rotating vortices are produced from shroud jet whose intensity reduces towards submerged entry nozzle (SEN) that can draw slag into the melt and adversely affect the non-metallic particle separation []. Also, the thinning of refractory linings of tundish by the role of shroud jet [3].

- Unstable vortices between the slag and melt are produced due to the rise of surface waves that can draw the slag into the melt [4].

This paper discusses the aspects of numerical methods in detail to avoid the above mentioned flow phenomenon and enhancing the tundish fluid flow.

\section{Mathematical Modelling}

The mathematical model study has been summed up under the following categories:

- Flow phenomenon: the coupled dense discrete phase model with realizable $\mathrm{k}-\varepsilon$ model and enhanced wall treatment was used to compute and simulate the fluid flow inside the tundish. During the teeming process, the tangential velocity above the nozzle will get stronger and stronger with the formation of a vortex. Li et al.[5] adopted the RNG (renormalization group) $\mathrm{k}-\varepsilon$ model and multiphase Volume of fluid (VOF) model to virtually derive the ladle teeming process, thus improving the rapid strained flow accuracy.

- Inclusion separation and transportation: Discrete phase model was adopted for inspecting the inclusion flotation.

- Residence time distribution: An unsteady species transport model using volumetric reactions with a diffusion energy source gave the specie concentration as a function of time. Eddy dissipation for the turbulence interaction converges the results to a stable solution.

- Adaptive meshing: Addition of finer mesh wherever needed to maintain smaller mesh size using dynamic isovalue adaption method. Often creating a mesh with an expanding cell volume that grows with the distance from the boundary, can lead to a problem where the mesh is not fine enough to resolve the flow, for the sake of avoiding a dense mesh. It can lead to adverse pressure gradient at the outlet or near the flow modifiers and make the flow reversible thus causing a major setback to our computed flow behavior. The region/volume based adaption feature is quite useful for refining regions that naturally require good resolution: for e.g., a blunt-body's wake region in a flow field. Also, we may use the region marking for creating registers of mask adaptation which can be further used to control the refinement and coarsening extent.

Fig. 1 shows the geometry as well as poly-hexcore 3D mesh used for the tundish geometry on which running numerical simulations were performed. Material properties are as illustrated in table 1 .

Table 1. Material properties

\begin{tabular}{|c|c|}
\hline Property & Value \\
\hline Density $\left(\mathrm{kg} / \mathrm{m}^{3}\right)$ & 7050 \\
\hline Thermal conductivity $(\mathrm{W} / \mathrm{m}-\mathrm{K})$ & 41 \\
\hline Specific heat $(\mathrm{J} / \mathrm{kg}-\mathrm{K})$ & 750 \\
\hline Dynamic viscosity $(\mathrm{kg} / \mathrm{m}-\mathrm{s})$ & 0.0067 \\
\hline
\end{tabular}

- To check the convergence during the solution, the average velocity in the spatial flow volume of the tundish can be also observed.

a.

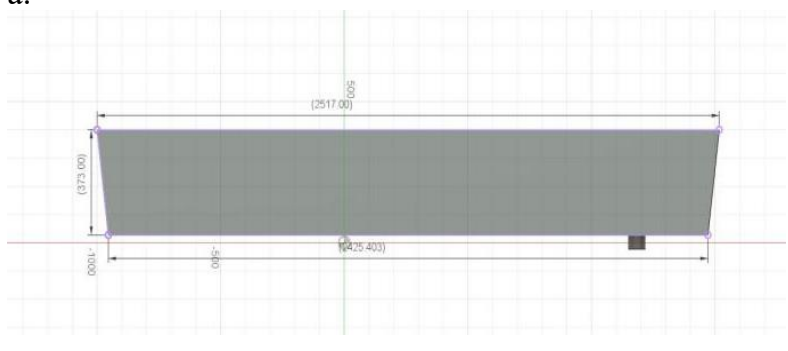


b.

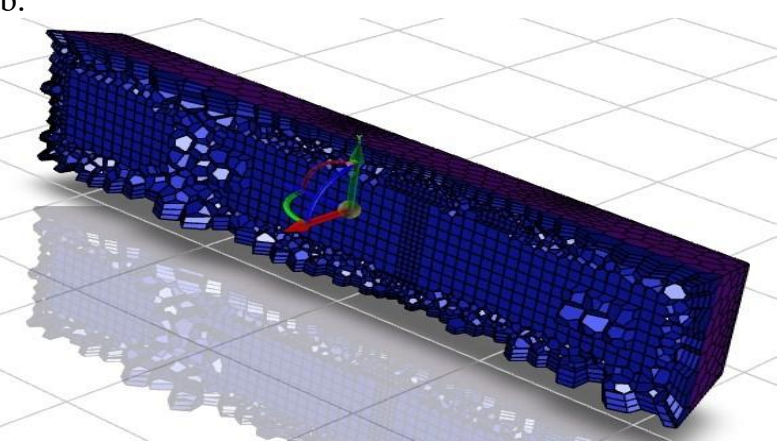

c.

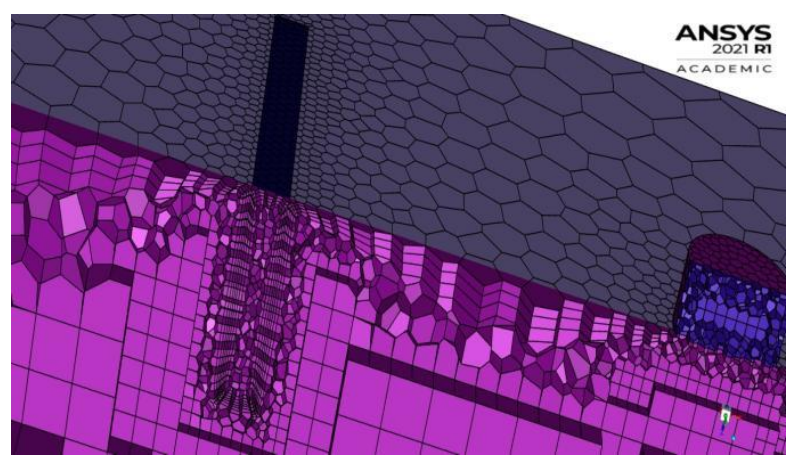

Fig. 1. (a)geometry, (b) 3-D poly hexcore meshed geometry with (c) finer mesh near the regions prone to velocity fluctuations which may lead to wall shear stress

\subsection{Governing Equations}

\subsubsection{Fluid flow phenomena}

Turbulence in a tundish has to be modelled under consideration of characteristic time and length. Eddy viscosity models are a class of turbulence models that are used to calculate Reynold's stresses. Only up to a particular length scale of spatial extension the turbulence is resolved. Large eddy simulations (LES) act as a bridge for connecting Direct Numerical Simulations (DNS) and the theoretical model. The Reynolds stress in the RANS equation (1) needs to be modelled to close the equation, as the Navier stokes contains all the information about the various force balances and momentum transfers on a fluid element:

$$
\begin{gathered}
\partial\left(\rho u_{i}\right) / \partial t+\nabla \\
\cdot\left(\rho u_{i} u_{j}\right)=-\nabla p+\nabla\left[\mu\left(\nabla u+(\nabla u)^{T}\right]\right. \\
+\rho g-\nabla\left(\frac{2}{3} \mu(\nabla u)\right)-\nabla\left(\rho \bar{u}_{i}^{i} \bar{u}_{j}^{\prime}\right)
\end{gathered}
$$

For the above equation, the most common approach for solving it is using the Bousinesqq hypothesis (2), i.e.

$$
\mu_{e f f}=\mu+\mu_{t}
$$

To calculate the value of eddy viscosity $\left(\boldsymbol{\mu}_{\boldsymbol{t}}\right)$ we need to use the k- $\varepsilon$ model. However, since we know that turbulence is both convected and diffused through the flow, and also it is not static or fixed at some distance from the wall, so we would like to solve transport equations for turbulent kinetic energy $(\mathrm{k})$ and turbulence dissipation rate $(\varepsilon)$. The transport equation for turbulent kinetic energy (3) is the same in the RNG, realizable and standard $\mathrm{k}-\varepsilon$ models, i.e.

$$
\begin{aligned}
\frac{\partial(\rho k)}{\partial t}+\nabla \cdot(\rho u k)= & \nabla\left[\left(\mu+\frac{\mu_{t}}{\sigma_{k}}\right) \nabla k\right]+P_{k}+P_{b} \\
& -\rho \epsilon+S_{k}
\end{aligned}
$$

Where the turbulent kinetic energy generation term due to diffusion in the fluid flow is represented by $\mathrm{T}$ as,

$$
T=\nabla\left[\left(\mu+\frac{\mu_{t}}{\sigma_{k}}\right) \nabla k\right]
$$

Whereas $\mathrm{P}_{\mathrm{k}}, \mathrm{P}_{\mathrm{b}}$, and $\mathrm{S}_{\mathrm{k}}$ represent production to mean velocity shear, buoyancy and user defined source, while $\rho \varepsilon$ acts as a sink in the equation.

The transport equation for dissipation rate (4) can also be represented as a scale determining equation corresponding to the size of the eddies in a $\mathrm{k}-\varepsilon$ model, i.e.

$$
\begin{aligned}
\frac{\partial(\rho \epsilon)}{\partial t}+\nabla \cdot(\rho u \epsilon)= & \nabla\left[\left(\mu+\frac{\mu_{t}}{\sigma_{\epsilon}}\right) \nabla \epsilon\right]-\frac{C_{2}}{k} \rho \epsilon^{2}+S_{\epsilon} \\
& +\frac{C_{1}}{k} \epsilon\left(P_{k}+C_{3} P_{b}\right)
\end{aligned}
$$

Empirical model coefficients $\mathrm{C}_{1}, \mathrm{C}_{2}$ and $\mathrm{C}_{3}$ vary depending upon the variant of $k-\varepsilon$ models we choose such as either standard, RNG or realizable k- $\varepsilon$ models. The Launder and Sharma (1974) model coefficients are most up to date and have been used here because of much better performance and accuracy as shown in table 2.

Table 2. Model coefficients as per Launder and Sharma

\begin{tabular}{|c|c|c|c|c|}
\hline $\mathrm{C}_{1 \mathrm{~s}}$ & $\mathrm{C}_{2 \mathrm{k}}$ & $\mathrm{C}_{\mu}$ & $\sigma_{\mathrm{k}}$ & $\sigma_{\mathrm{k}}$ \\
\hline 1.44 & 1.92 & 0.09 & 1.0 & 1.3 \\
\hline
\end{tabular}

Once the transport equations for $\mathrm{k}$ and $\varepsilon$ have been solved we can compute $\boldsymbol{\mu}_{t}$ as,

$$
\mu_{t}=\frac{C_{\mu} \rho k^{2}}{\epsilon}
$$

Now, on putting (5) in Bousinesqq hypothesis equation (2) and then in Reynold's Average Navier Stokes (RANS) equation (1) we can close the system. It should be noted that $\mathrm{C} \mu$ is a function of the velocity gradients in the realizable k-e model .

\subsubsection{Inclusion separation and transportation}

The discrete phase model was used with the lagrangianeulerian multiphase approach for inclusion particle tracking. With consideration of fluid as a continuum, the dispersed phase is solved using the lagrangian approach with multiparticle representation and Liouville equation, while the carrier phase is solved using the the RANS equations and principle of large eddy simulations. An assumption made in this model is that the secondary dispersed phase occupies a lesser volume 
fraction as the dispersed phase exchanges momentum, energy and mass with the fluid phase irrespective of high mass condition that is virtually valid, i.e.

$$
\dot{m}_{\text {particles }} \geq \dot{m}_{\text {fluid }}
$$

During the fluid phase calculation, the inclusion trajectories are computed separately at specific intervals using the transport equation (8) for inclusion movement as,

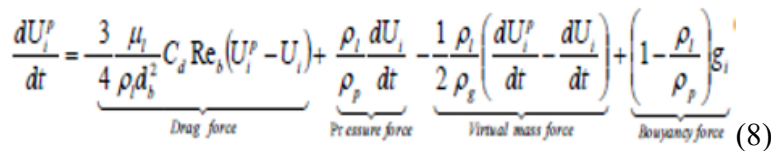

\subsubsection{Residence time distribution}

The probability distribution of time at which the mixed fluid stays inside the controlled flow volume is generally termed as the residence time distribution. RTD is nothing but a normalized response of the system to an impulse stimulant injection in the form of $\delta$-dirac distribution. Conventionally, as the injection of a tracer from the inlet into the system begins, its weighted-area average concentration is measured w.r.t time at the inlet as well as the outlet which is then further used to determine the residence time distribution. But now, with the use of unsteady species transport model where a tracer is modelled as species in a user-defined scalar equation, and also with the help of finite volume method, it was possible to solve partial differential equations from the conservation laws. At outlet, the surface area-averaged tracer concentration was closely monitored as a function of time. Usually, convection is followed up by diffusion in these kinds of processes and hence we have considered a combined effect of diffusion and convection. The residence time distribution function $\mathrm{E}(\mathrm{t})$ depicts in a quantitative manner how much time is spent by different fluid elements in the controlled volume, i.e. in a continuous process system it gives data on any defects in the fluid flow pattern, i.e. recirculation and mixing.

The general transport equation (9) for the combined convective-diffusion process is,

$$
\frac{\partial(\rho m)}{\partial t}+\operatorname{div}(\rho m u)=\operatorname{div}(\Gamma \operatorname{grad}(m))+S_{m}
$$

\subsection{Boundary Conditions}

There is a no-slip condition at the walls of the tundish and zero normal velocity gradients on the liquid steel free surface. With 5\% turbulence intensity and hydraulic diameter inter-relations, $\mathrm{k}$ and $\varepsilon$ are assigned in the interior. For inclusion travel, particles are permitted to reflect from the tundish walls but made to escape from the outlet. Rest other parameters are as shown below:

\begin{tabular}{|c|c|}
\hline Inlet Condition & Velocity inlet \\
\hline Inlet temperature & $1773 \mathrm{~K}$ \\
\hline Heat flux at free surface of tundish & $13 \mathrm{KW} / \mathrm{m}^{2}$ \\
\hline Heat loss at bottom wall of tundish & $1.7 \mathrm{KW} / \mathrm{m}^{2}$ \\
\hline Heat loss at long wall of tundish & $3.6 \mathrm{KW} / \mathrm{m}^{2}$ \\
\hline Heat loss at short wall of tundish & $3.98 \mathrm{KW} / \mathrm{m}^{2}$ \\
\hline
\end{tabular}

\subsection{Numerical Solutions}

The governing turbulence, flow and associated transport equations are solved using various numerical methods. Using the PISO algorithm was quite a beneficial coupling of the velocity and pressure terms assuring the convergence efficiency. To avoid drastic abrupt changes in high swirl and internal pressure, PRESTO pressure staggering option. The coupled 3-D, non-linear behavioral partial differential equations of momentum, turbulent kinetic energy and turbulent dissipation rate were solved using second order upwind discretization. The convergence criterion was 1e-04, was fixed for all equations except RTD where it was of the value 1e-10. With a time step of $0.01 \mathrm{~s}$ and running 20 iterations per time step all the calculation was done in ANSYS FLUENT solver.

\section{Results and discussions}

\subsection{Analysis of fluid flow}

Fig. 2. illustrates the velocity profile contour for the optimized proposed model from the existing tundish. It was closely observed that there was significant turbulence near the inlet. Due to presence of turbo furniture, there was a few striking backs of the flow towards the inlet while the rest were dispersed throughout the flow volume. The significance of tundish furniture can be much appreciated here as we can clearly observe that it nearly suppresses the splashing effect when there is an initial impact of liquid steel. Also, with the impact of liquid steel on the walls of furniture, it deeply reduces the chances of eddy formation and any vorticities near the outside region of the tundish furniture. After the tundish furniture is filled up and with the rise in liquid level inside the tundish, the wave formations at the free surface start coming to a normal, stable and calm condition because of the application of a turbo stopper. From fig. 3. we can also observe the occurrence of few dead zones due to spatial limitation to the turbulent flow that causes deceleration of jet as the flow moves upward after striking the furniture and with an increase in the liquid level. 


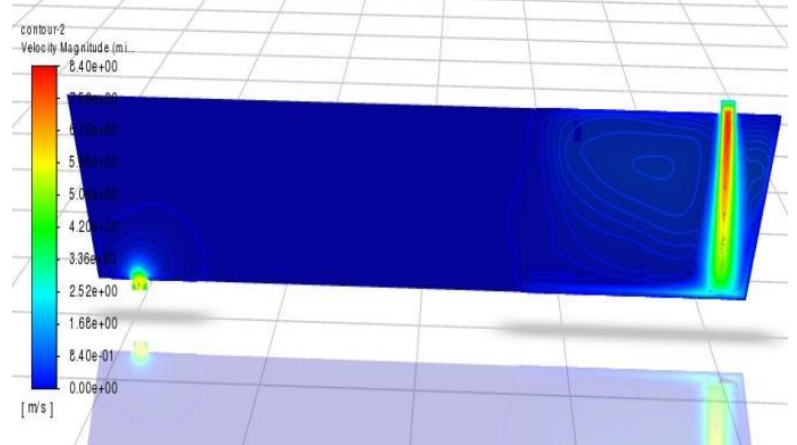

Fig. 2. The velocity profile contour showing turbulence at the inlet region up to the turbo stopper that makes the fluid flow stable and also contributes to a homogenous flow

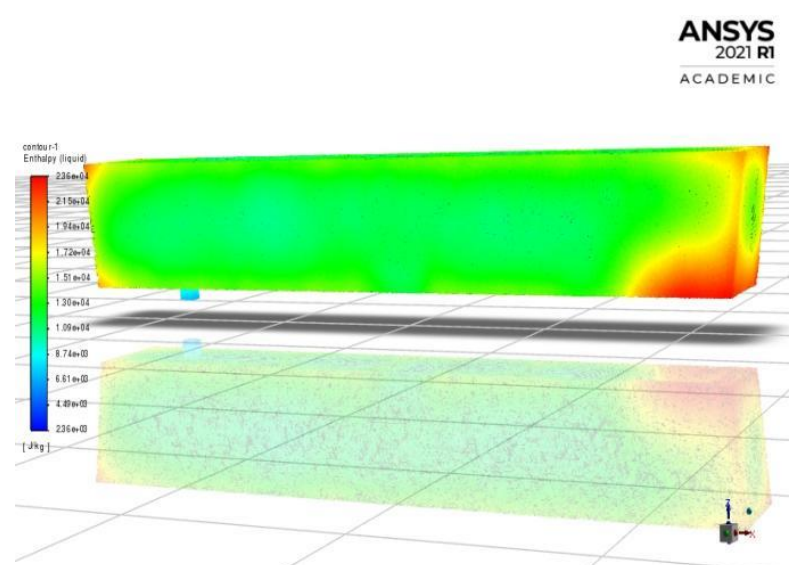

Fig. 3. The dead zones formed inside the fluid flow volume of the existing tundish

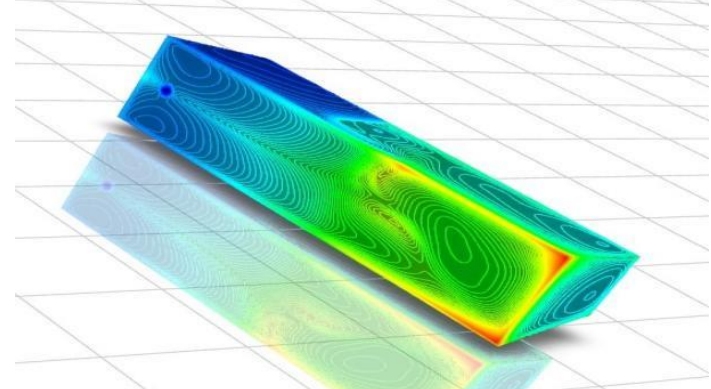

Fig. 4. Reduced dead zones from the optimized model

Also, from fig. 5. we can also observe the slag formation near the end of our iterations of filling up of the tundish. The temperature distribution profile on the fluid flow volume inside the tundish shows the phenomenon of deposition of slag at the free surface of the liquid that performs various functions, such as preventing the rest of the liquid steel below to get oxidised and as a good inclusion adsorber. The flow's stability is depicted by fig. 6 . in which the fluid particle trajectories linear behavior after the dams before the outflow from the outlet is shown that correlates with the stability of our fluid. The stability of our fluid flow can also be validated from fig. 7. which illustrates the pathlines of the fluid particles showing no intersection and vorticities inside the fluid flow region.

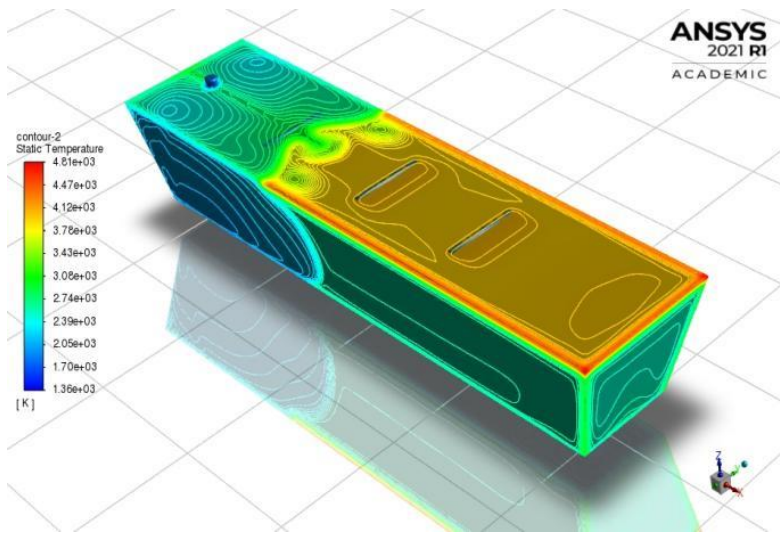

Fig. 5. The temperature distribution profile of the fluid flow volume showing the phenomenon of slab formation near the free surface

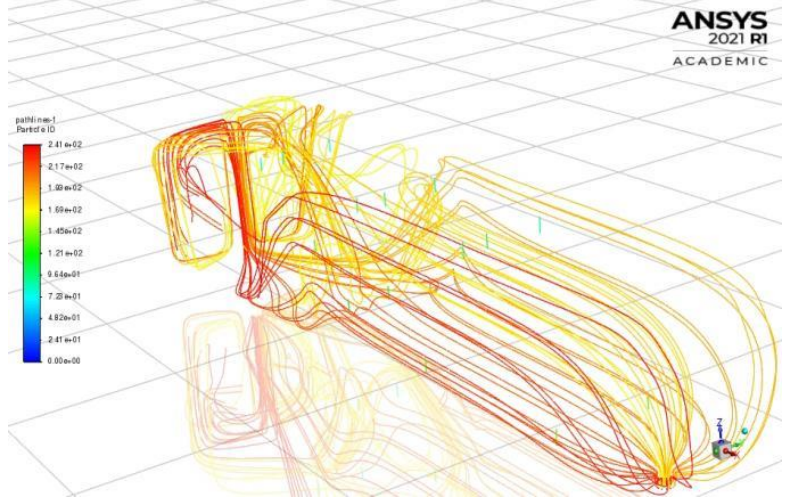

Fig. 6. The linear particle trajectories after crossing the flow modulators (weirs) before exiting from the outlet in the flow volume

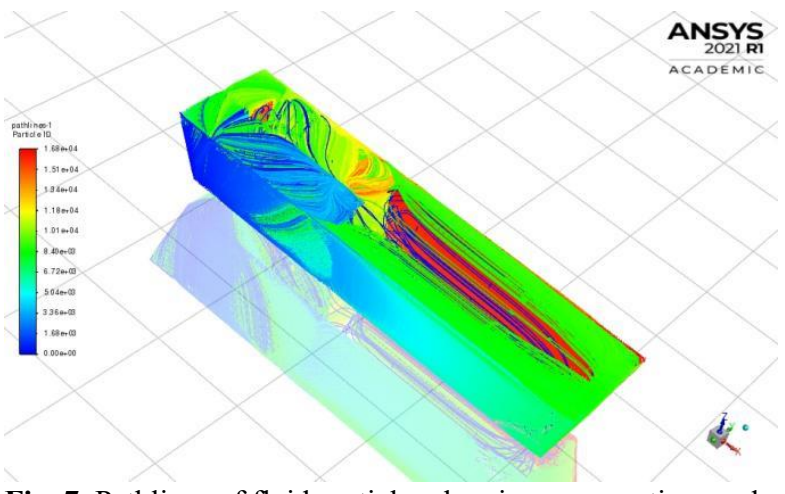

Fig. 7. Pathlines of fluid particles showing zero vortices and no intersection with a better homogenous fluid flow.

\subsection{Analysis of Inclusion Transportation}

Inclusion can be seen in fig. 8. inside the tundish flow volume. These non-metallic inclusions can be seen moving towards the free surface after impact from the flow modulators (dams and weirs). The strategic placements of these dams help in the separation of inclusions and make their travel to the free surface where slag is already present that eventually captures these inclusions and contribute to the production of high quality steel. 


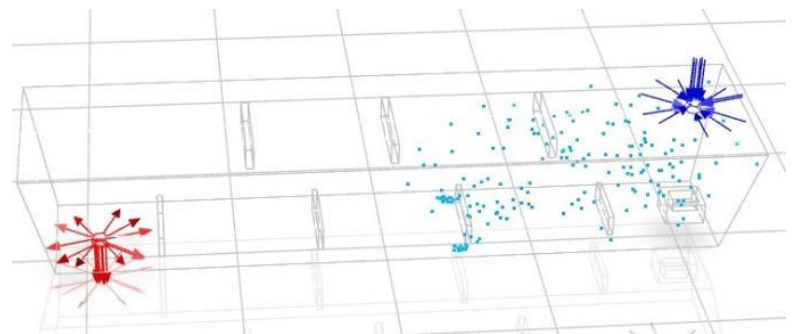

Fig. 8. Non-metallic Inclusion flotation towards the free surface can be seen represented with blue coloured particles

\subsection{Residence time distribution}

Fig. 9. shows the residence time distribution curve depicting the species concentration as a function of time. Here, three cases of one with turbo stopper only, the second one having a turbo stopper and 2 dams in the tundish design and the third case with a turbo stopper and 4 dams in the tundish design. Increased residence time promotes more time for the inclusions to travel from the molten liquid to the free surface, thus promoting more non-metallic inclusions to get entrapped into the slag. It also helped in the dead areas to stop the process of skull formation. These factors contribute to the good intermixing and homogenization of liquid metal.

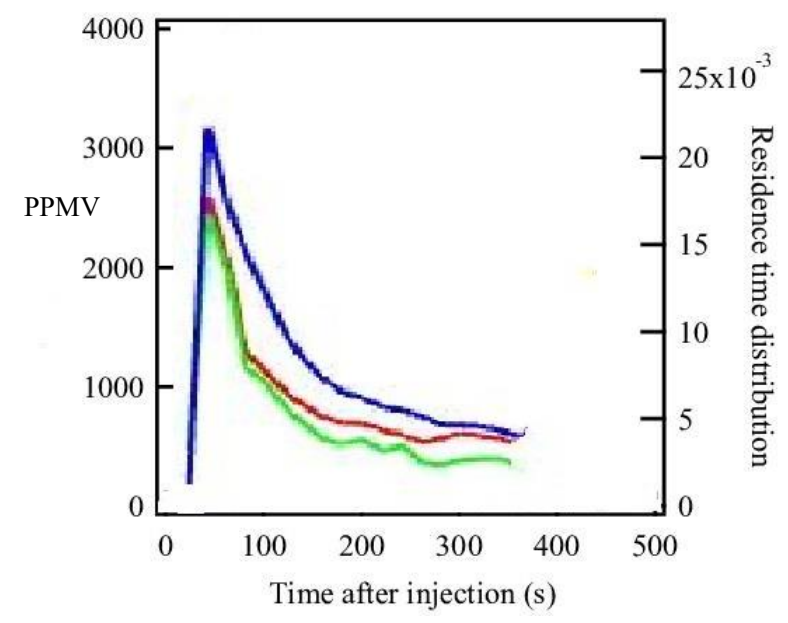

Fig. 9. Residence time distribution showing species concentration as a function of time.

\section{Conclusion}

This paper discussed the fluid flow phenomenon inside the tundish with an optimized design and simulation using a multiphase mathematical model using the eulerian approach dense discrete phase method, RNG k$\varepsilon$ model with enhanced wall treatment. This model was further used to exhibit the residence time distribution and to also display the inclusion flotation using the unsteady species transport model. Further investigations on the ladle change phenomenon of the transient state will be discussed in upcoming research works.
1. US5358551 Patent, Turbulence inhibiting tundish and impact pad and method of using (1994).

2. US5131635 Patent, Impact pad with rising flow surface (1992).

3. Odenthal H.-J., Bölling R., Pfeifer H., $2^{\text {nd }}$ International Conference on the Science and Technology of Steelmaking, 499, Swansea, UK (2001).

4. H. J. Odenthal, R. Bölling, H. Pfeifer, J. F Holzhauser, F.J. Wahlers, Steel Research, 72, 466 (2001).

5. H.X. Li, Q. Wang, H. Lei, J.W. Jiang, Z.C. Guo, J.C. He, ISIJ International, 54, 1592 (2014).

\section{References}

